\title{
Research Article \\ Generic Lightlike Submanifolds of an Indefinite Cosymplectic Manifold
}

\author{
Dae Ho Jin' ${ }^{1}$ and Jae Won Lee ${ }^{2}$ \\ ${ }^{1}$ Department of Mathematics, Dongguk University, Gyeongju 780-714, Republic of Korea \\ ${ }^{2}$ Department of Mathematics, Sogang University, Sinsu-dong, Mapo-gu 121-742, Republic of Korea
}

Correspondence should be addressed to Jae Won Lee, leejaewon@sogang.ac.kr

Received 7 June 2011; Revised 12 August 2011; Accepted 2 September 2011

Academic Editor: Gerhard-Wilhelm Weber

Copyright (c) 2011 D. H. Jin and J. W. Lee. This is an open access article distributed under the Creative Commons Attribution License, which permits unrestricted use, distribution, and reproduction in any medium, provided the original work is properly cited.

Lightlike geometry has its applications in general relativity, particularly in black hole theory. Indeed, it is known that lightlike hypersurfaces are examples of physical models of Killing horizons in general relativity (Galloway, 2007). In this paper, we introduce the definition of generic lightlike submanifolds of an indefinite cosymplectic manifold. We investigate new results on a class of generic lightlike submanifolds $M$ of an indefinite cosymplectic manifold $\bar{M}$.

\section{Introduction}

In the generalization from Riemannian to semi-Riemannian manifolds, the induced metric may be degenerate (lightlike) therefore there is a natural existence of lightlike submanifolds and for which the local and global geometry is completely different than nondengerate case. In lightlike case, the standard textbook definitions do not make sense- and one fails to use the theory of non-degenerate geometry in the usual way. The primary difference between the lightlike submanifolds and non-degenerate submanifolds is that in the first case, the normal vector bundle intersects with the tangent bundle. Thus, the study of lightlike submanifolds becomes more difficult and different from the study of non-degenerate submanifolds. Moreover, the geometry of lightlike submanifolds is used in mathematical physics, in particular, in general relativity since lightlike submanifolds produce models of different types of horizons (event horizons, Cauchy's horizons, and Kruskal's horizons). The universe can be represented as a four-dimensional submanifold embedded in a $(4+n)$-dimensional spacetime manifold. Lightlike hypersurfaces are also studied in the thoery of electromagnetism [1]. Thus, large number of applications but limited information available, motivated us to do research on this subject matter. Kupeli [2] and Bejancu and Duggal [1] developed the general 
theory of degenerate (lightlike) submanifolds. They constructed a transversal vector bundle of lightlike submanifold and investigated various properties of these manifolds. The geometry of both lightlike hypersurfaces and half lightlike submanifolds of indefinite cosymplectic manifolds was studied by Jin $([3,4])$. However, a general notion of generic lightlike submanifolds of an indefinite cosymplectic manifold has not been introduced as yet.

The objective of this paper is to study generic $r$-lightlike submanifolds $M$ of an indefinite cosymplectic manifold $\bar{M}$ subject to the conditions: (1) $M$ is totally umbilical, or (2) $S(T M)$ is totally umbilical in $M$. In Section 1, we first of all recall some of fundamental formulas in the theory of $r$-lightlike submanifolds. In Section 2, we newly define generic lightlike submanifolds. After that, we prove some basic theorems which will be used in the rest of this paper. In Section 3, we study generic $r$-lightlike submanifolds of $\bar{M}$.

\section{Lightlike Submanifolds}

Let $(M, g)$ be an $m$-dimensional lightlike submanifold of an $(m+n)$-dimensional semiRiemannian manifold $(\bar{M}, \bar{g})$. Then the radical distribution $\operatorname{Rad}(T M)=T M \cap T M^{\perp}$ is a vector subbundle of the tangent bundle $T M$ and the normal bundle $T M^{\perp}$, of rank $r(1 \leq$ $r \leq \min \{m, n\})$. In general, there exist two complementary non-degenerate distributions $S(T M)$ and $S\left(T M^{\perp}\right)$ of $\operatorname{Rad}(T M)$ in $T M$ and $T M^{\perp}$, respectively, called the screen and coscreen distributions on $M$, such that

$$
T M=\operatorname{Rad}(T M) \oplus_{\text {orth }} S(T M), \quad T M^{\perp}=\operatorname{Rad}(T M) \oplus_{\text {orth }} S\left(T M^{\perp}\right),
$$

where the symbol $\oplus_{\text {orth }}$ denotes the orthogonal direct sum. We denote such a lightlike submanifold by $\left(M, g, S(T M), S\left(T M^{\perp}\right)\right)$. Denote by $F(M)$ the algebra of smooth functions on $M$ and by $\Gamma(E)$ the $F(M)$ module of smooth sections of a vector bundle $E$ over $M$. We use the same notation for any other vector bundle. We use the following range of indices:

$$
i, j, k, \ldots \in\{1, \ldots, r\}, \quad \alpha, \beta, \gamma, \ldots \in\{r+1, \ldots, n\}
$$

Let $\operatorname{tr}(T M)$ and $\operatorname{ltr}(T M)$ be complementary (but not orthogonal) vector bundles to $T M$ in $T \bar{M}_{\mid M}$ and $T M^{\perp}$ in $S(T M)^{\perp}$, respectively, and let $\left\{N_{1}, \ldots, N_{r}\right\}$ be a lightlike basis of

$\Gamma\left(\operatorname{ltr}(T M)_{\mid \mathfrak{u}}\right)$ consisting of smooth sections of $S(T M)_{\left.\right|_{\mathfrak{u}}}^{\perp}$, where $\mathcal{U}$ is a coordinate neighborhood of $M$, such that

$$
\bar{g}\left(N_{i}, \xi_{j}\right)=\delta_{i j}, \quad \bar{g}\left(N_{i}, N_{j}\right)=0,
$$

where $\left\{\xi_{1}, \ldots, \xi_{r}\right\}$ is a lightlike basis of $\Gamma(\operatorname{Rad}(T M))$. Then we have

$$
\begin{aligned}
T \bar{M} & =T M \oplus \operatorname{tr}(T M)=\{\operatorname{Rad}(T M) \oplus \operatorname{tr}(T M)\} \oplus_{\text {orth }} S(T M) \\
& =\{\operatorname{Rad}(T M) \oplus \operatorname{ltr}(T M)\} \oplus_{\text {orth }} S(T M) \oplus_{\text {orth }} S\left(T M^{\perp}\right)
\end{aligned}
$$


We say that a lightlike submanifold $\left(M, g, S(T M), S\left(T M^{\perp}\right)\right)$ of $\bar{M}$ is

(1) $r$-lightlike if $1 \leq r<\min \{m, n\}$;

(2) coisotropic if $1 \leq r=n<m$;

(3) isotropic if $1 \leq r=m<n$;

(4) totally lightlike if $1 \leq r=m=n$.

The above three classes (2)-(4) are particular cases of the class (1) as follows: $S\left(T M^{\perp}\right)=$ $\{0\}, S(T M)=\{0\}$, and $S(T M)=S\left(T M^{\perp}\right)=\{0\}$, respectively. The geometry of $r$-lightlike submanifolds is more general form than that of the other three type submanifolds. For this reason, in this paper we consider only $r$-lightlike submanifolds $M \equiv\left(M, g, S(T M), S\left(T M^{\perp}\right)\right)$, with the following local quasiorthonormal field of frames of $\bar{M}$ :

$$
\left\{\xi_{1}, \ldots, \xi_{r}, N_{1}, \ldots, N_{r}, F_{r+1}, \ldots, F_{m}, E_{r+1}, \ldots, E_{n}\right\},
$$

where the sets $\left\{F_{r+1}, \ldots, F_{m}\right\}$ and $\left\{E_{r+1}, \ldots, E_{n}\right\}$ are orthonormal basis of $\Gamma(S(T M))$ and $\Gamma\left(S\left(T M^{\perp}\right)\right)$, respectively.

Let $\bar{\nabla}$ be the Levi-Civita connection of $\bar{M}$ and $P$ the projection morphism of $\Gamma(T M)$ on $\Gamma(S(T M))$ with respect to (2.1). For an $r$-lightlike submanifold, the local Gauss-Weingarten formulas are given by

$$
\begin{aligned}
\bar{\nabla}_{X} Y & =\nabla_{X} Y+\sum_{i=1}^{r} h_{i}^{\ell}(X, Y) N_{i}+\sum_{\alpha=r+1}^{n} h_{\alpha}^{s}(X, Y) E_{\alpha,} \\
\bar{\nabla}_{X} N_{i} & =-A_{N_{i}} X+\sum_{j=1}^{r} \tau_{i j}(X) N_{j}+\sum_{\alpha=r+1}^{n} \rho_{i \alpha}(X) E_{\alpha,} \\
\bar{\nabla}_{X} E_{\alpha} & =-A_{E_{\alpha}} X+\sum_{i=1}^{r} \phi_{\alpha i}(X) N_{i}+\sum_{\beta=r+1}^{n} \sigma_{\alpha \beta}(X) E_{\beta}, \\
\nabla_{X} P Y & =\nabla_{X}^{*} P Y+\sum_{i=1}^{r} h_{i}^{*}(X, P Y) \xi_{i}, \\
\nabla_{X} \xi_{i} & =-A_{\xi_{i}}^{*} X-\sum_{j=1}^{r} \tau_{j i}(X) \xi_{j},
\end{aligned}
$$

for any $X, Y \in \Gamma(T M)$, where $\nabla$ and $\nabla^{*}$ are induced linear connections on $T M$ and $S(T M)$, respectively, the bilinear forms $h_{i}^{\ell}$ and $h_{\alpha}^{s}$ on $M$ are called the local lightlike and screen second fundamental forms on $T M$, respectively, $h_{i}^{*}$ are called the local radical second fundamental forms on $S(T M) . A_{N_{i}}, A_{\xi_{i}}^{*}$ and $A_{E_{\alpha}}$ are linear operators on $\Gamma(T M)$ and $\tau_{i j}, \rho_{i \alpha}, \phi_{\alpha i}$ and $\sigma_{\alpha \beta}$ are 1forms on $T M$. Since $\bar{\nabla}$ is torsion-free, $\nabla$ is also torsion-free and both $h_{i}^{\ell}$ and $h_{\alpha}^{s}$ are symmetric. From the fact $h_{i}^{\ell}(X, Y)=\bar{g}\left(\bar{\nabla}_{X} Y, \xi_{i}\right)$, we know that $h_{i}^{\ell}$ are independent of the choice of a screen distribution. We say that

$$
h(X, Y)=\sum_{i=1}^{r} h_{i}^{\ell}(X, Y) N_{i}+\sum_{\alpha=r+1}^{n} h_{\alpha}^{s}(X, Y) E_{\alpha}
$$

is the second fundamental tensor of $M$. 
The induced connection $\nabla$ on $T M$ is not metric and satisfies

$$
\left(\nabla_{X} g\right)(Y, Z)=\sum_{i=1}^{r}\left\{h_{i}^{\ell}(X, Y) \eta_{i}(Z)+h_{i}^{\ell}(X, Z) \eta_{i}(Y)\right\}
$$

for all $X, Y \in \Gamma(T M)$, where $\eta_{i} s$ are the 1 -forms such that

$$
\eta_{i}(X)=\bar{g}\left(X, N_{i}\right), \quad \forall X \in \Gamma(T M)
$$

But the connection $\nabla^{*}$ on $S(T M)$ is metric. The above three local second fundamental forms are related to their shape operators by

$$
\begin{aligned}
h_{i}^{\ell}(X, Y) & =g\left(A_{\xi_{i}}^{*} X, Y\right)-\sum_{k=1}^{r} h_{k}^{\ell}\left(X, \xi_{i}\right) \eta_{k}(Y), \\
h_{i}^{\ell}(X, P Y) & =g\left(A_{\xi_{i}}^{*} X, P Y\right), \quad \bar{g}\left(A_{\xi_{i}}^{*} X, N_{j}\right)=0, \\
\epsilon_{\alpha} h_{\alpha}^{s}(X, Y) & =g\left(A_{E_{\alpha}} X, Y\right)-\sum_{i=1}^{r} \phi_{\alpha i}(X) \eta_{i}(Y), \\
\epsilon_{\alpha} h_{\alpha}^{s}(X, P Y) & =g\left(A_{E_{\alpha}} X, P Y\right), \quad \bar{g}\left(A_{E_{\alpha}} X, N_{i}\right)=\epsilon_{\alpha} \rho_{i \alpha}(X), \\
h_{i}^{*}(X, P Y) & =g\left(A_{N_{i}} X, P Y\right), \quad \eta_{j}\left(A_{N_{i}} X\right)+\eta_{i}\left(A_{N_{j}} X\right)=0, \\
\epsilon_{\beta} \sigma_{\alpha \beta} & =-\epsilon_{\alpha} \sigma_{\beta \alpha}, \quad \forall X, Y \in \Gamma(T M),
\end{aligned}
$$

where $\epsilon_{\alpha}=\bar{g}\left(E_{\alpha}, E_{\alpha}\right)(= \pm 1)$ is the sign of the vector field $E_{\alpha}$. From (2.18), we know that each $A_{N_{i}}$ is shape operator related to the local second fundamental form $h_{i}^{*}$ on $S(T M)$. Replace $Y$ by $\xi_{j}$ in (2.14), we have

$$
h_{i}^{\ell}\left(X, \xi_{j}\right)+h_{j}^{\ell}\left(X, \xi_{i}\right)=0
$$

for all $X \in \Gamma(T M)$. It follows

$$
h_{i}^{\ell}\left(X, \xi_{i}\right)=0, \quad h_{i}^{\ell}\left(\xi_{j}, \xi_{k}\right)=0
$$

Also, replace $X$ by $\xi_{j}$ in (2.14) and use (2.21), we have

$$
h_{i}^{\ell}\left(X, \xi_{j}\right)=g\left(X, A_{\xi_{i}}^{*} \xi_{j}\right), \quad A_{\xi_{i}}^{*} \xi_{j}+A_{\xi_{j}}^{*} \xi_{i}=0, \quad A_{\xi_{i}}^{*} \xi_{i}=0
$$

For an $r$-lightlike submanifold, replace $Y$ by $\xi_{i}$ in (2.16), we have

$$
h_{\alpha}^{s}\left(X, \xi_{i}\right)=-\epsilon_{\alpha} \phi_{\alpha i}(X), \quad \forall X \in \Gamma(T M) .
$$


From (2.6), (2.10), and (2.23), for all $X \in \Gamma(T M)$, we have

$$
\bar{\nabla}_{X} \xi_{i}=-A_{\xi_{i}}^{*} X-\sum_{j=1}^{r} \tau_{j i}(X) \xi_{j}-\sum_{\alpha=r+1}^{n} \epsilon_{\alpha} \phi_{\alpha i}(X) E_{\alpha}+\sum_{j=1}^{r} h_{j}^{\ell}\left(X, \xi_{i}\right) N_{j}
$$

Definition 2.1. A lightlike submanifold $M$ of a semi-Riemannian manifold $(\bar{M}, \bar{g})$ is said to be irrotational if $\bar{\nabla}_{X} \xi_{i} \in \Gamma(T M)$ for any $X \in \Gamma(T M)$ and $\xi_{i} \in \Gamma(\operatorname{Rad}(T M))$ for all $i$.

Note 1. For an $r$-lightlike $M$, the above definition is equivalent to

$$
h_{j}^{\ell}\left(X, \xi_{i}\right)=0, \quad h_{\alpha}^{s}\left(X, \xi_{i}\right)=\phi_{\alpha i}(X)=0, \quad \forall X \in \Gamma(T M)
$$

Denote by $\bar{R}$ and $R$ the curvature tensors of $\bar{\nabla}$ and $\nabla$, respectively. Using the local Gauss-Weingarten formulas for $M$, we obtain

$$
\begin{aligned}
& \bar{R}(X, Y) Z=R(X, Y) Z \\
& +\sum_{i=1}^{r}\left\{h_{i}^{\ell}(X, Z) A_{N_{i}} Y-h_{i}^{\ell}(Y, Z) A_{N_{i}} X\right\} \\
& +\sum_{\alpha=r_{1}}^{n}\left\{h_{\alpha}^{s}(X, Z) A_{E_{\alpha}} Y-h_{\alpha}^{s}(Y, Z) A_{E_{\alpha}} X\right\} \\
& +\sum_{i=1}^{r}\left\{\left(\nabla_{X} h_{i}^{\ell}\right)(Y, Z)-\left(\nabla_{Y} h_{i}^{\ell}\right)(X, Z)\right. \\
& +\sum_{j=1}^{r}\left[\tau_{j i}(X) h_{j}^{\ell}(Y, Z)-\tau_{j i}(Y) h_{j}^{\ell}(X, Z)\right] \\
& \left.+\sum_{\alpha=r+1}^{n}\left[\phi_{\alpha i}(X) h_{\alpha}^{s}(Y, Z)-\phi_{\alpha i}(Y) h_{\alpha}^{s}(\mathrm{X}, Z)\right]\right\} N_{i} \\
& +\sum_{\alpha=r+1}^{n}\left\{\left(\nabla_{X} h_{\alpha}^{s}\right)(Y, Z)-\left(\nabla_{Y} h_{\alpha}^{s}\right)(X, Z)\right. \\
& +\sum_{i=1}^{r}\left[\rho_{i \alpha}(X) h_{i}^{\ell}(Y, Z)-\rho_{i \alpha}(Y) h_{\alpha}^{s}(X, Z)\right] \\
& \left.+\sum_{\beta=r+1}^{n}\left[\sigma_{\beta \alpha}(X) h_{\beta}^{s}(Y, Z)-\sigma_{\beta \alpha}(Y) h_{\beta}^{s}(X, Z)\right]\right\} E_{\alpha,}
\end{aligned}
$$


for all $X, Y, Z \in \Gamma(T M)$. Assume that $M$ is irrotational. Replace $Z$ by $\xi_{k}$ in (2.26) and use (2.10), (2.15), (2.17), and (2.25), then we have

$$
\begin{aligned}
\bar{R}(X, Y) \xi_{k}= & R(X, Y) \xi_{k} \\
& +\sum_{i=1}^{r}\left\{g\left(A_{\xi_{i}}^{*} Y, A_{\xi_{k}}^{*} X\right)-g\left(A_{\xi_{i}}^{*} X, A_{\xi_{k}}^{*} Y\right)\right\} N_{i} \\
& +\sum_{\alpha=r+1}^{n} \epsilon_{\alpha}\left\{g\left(A_{E_{\alpha}} Y, A_{\xi_{k}}^{*} X\right)-g\left(A_{E_{\alpha}} X, A_{\xi_{k}}^{*} Y\right)\right\} E_{\alpha} .
\end{aligned}
$$

Using (2.27) and the fact $R(X, Y) Z \in \Gamma(T M)$ for $X, Y, Z \in \Gamma(T M)$, we get

$$
\begin{aligned}
\bar{g}\left(\bar{R}(X, Y) Z, \xi_{k}\right) & =-\bar{g}\left(\bar{R}(X, Y) \xi_{k}, Z\right) \\
& =-g\left(R(X, Y) \xi_{k}, Z\right)+\sum_{i=1}^{r}\left\{g\left(A_{\xi_{i}}^{*} X, A_{\xi_{k}}^{*} Y\right)-g\left(A_{\xi_{i}}^{*} Y, A_{\xi_{k}}^{*} X\right)\right\} \eta_{i}(Z) \\
& =g\left(R(X, Y) Z, \xi_{k}\right)+\sum_{i=1}^{r}\left\{g\left(A_{\xi_{i}}^{*} X, A_{\xi_{k}}^{*} Y\right)-g\left(A_{\xi_{i}}^{*} Y, A_{\xi_{k}}^{*} X\right)\right\} \eta_{i}(Z) \\
& =\sum_{i=1}^{r}\left\{g\left(A_{\xi_{i}}^{*} X, A_{\xi_{k}}^{*} Y\right)-g\left(A_{\xi_{i}}^{*} Y, A_{\xi_{k}}^{*} X\right)\right\} \eta_{i}(Z), \quad \forall X, Y, Z \in \Gamma(T M) .
\end{aligned}
$$

\section{Indefinite Cosymplectic Manifolds}

An odd dimensional smooth manifold $(\bar{M}, \bar{g})$ is called a contact metric manifold $[5,6]$ if there exists a $(1,1)$-type tensor field $J$, a vector field $\zeta$, called the characteristic vector field, and its 1 -form $\theta$ satisfying

$$
\begin{aligned}
& J^{2} X=-X+\theta(X) \zeta, \quad J \zeta=0, \quad \theta \circ J=0, \quad \theta(\zeta)=1, \\
& \bar{g}(\zeta, \zeta)=\epsilon, \quad \bar{g}(J X, J Y)=\bar{g}(X, Y)-\epsilon \theta(X) \theta(Y), \\
& \theta(X)=\epsilon \bar{g}(\zeta, X), \quad d \theta(X, Y)=\bar{g}(J X, Y), \quad \epsilon= \pm 1,
\end{aligned}
$$

for any vector fields $X, Y$ on $\bar{M}$. Then the set $(J, \theta, \zeta, \bar{g})$ is called a contact metric structure on $\bar{M}$. Note that we may assume that $\epsilon=1$ without loss of generality [7]. We say that $\bar{M}$ has a normal contact structure $[5,8]$ if $N_{J}+d \theta \otimes \zeta=0$, where $N_{J}$ is the Nijenhuis tensor field of $J$. A normal contact metric manifold is called a cosymplectic $[9,10]$ for which we have

$$
\bar{\nabla}_{X} \theta=0, \quad \bar{\nabla}_{X} J=0,
$$

for any vector field $X$ on $\bar{M}$. A cosymplectic manifold $\bar{M}=(\bar{M}, J, \zeta, \theta, \bar{g})$ is called an indefinite cosymplectic manifold $[3,4]$ if $(\bar{M}, \bar{g})$ is a semi-Riemannian manifold of index $\mu(>0)$. For any 
indefinite cosymplectic manifold, apply $\bar{\nabla}_{X}$ to $J \zeta=0$ for any vector field $X$ on $\bar{M}$ and use (3.2), then we have $J\left(\bar{\nabla}_{X} \zeta\right)=0$. Apply $J$ to this and use (3.1) and $\theta\left(\bar{\nabla}_{X} \zeta\right)=0$, we get

$$
\bar{\nabla}_{X} \zeta=0
$$

An indefinite cosymplectic manifold $\bar{M}$ is called an indefinite cosymplectic space form, denoted by $\bar{M}(c)$, if it has the constant $J$-sectional curvature $c[3,9,10]$. The curvature tensor $\bar{R}$ of this space form $\bar{M}(c)$ is given by

$$
\begin{aligned}
\bar{R}(X, Y) Z=\frac{c}{4}\{\bar{g}(Y, Z) X-\bar{g}(X, Z) Y+\theta(X) \theta(Z) Y \\
-\theta(Y) \theta(Z) X+\bar{g}(X, Z) \theta(Y) \zeta-\bar{g}(Y, Z) \theta(X) \zeta \\
+\bar{g}(J Y, Z) J X+\bar{g}(J Z, X) J Y-2 \bar{g}(J X, Y) J Z\}
\end{aligned}
$$

for any vector fields $X, Y$, and $Z$ in $\bar{M}$.

Let $M$ be an $m$-dimensional $r$-lightlike submanifold of an $(m+n)$-dimensional indefinite cosymplectic manifold $\bar{M}$ and $P$ the projection morphism of $\Gamma(T M)$ on $\Gamma(S(T M))$ with respect to (2.4). The characteristic vector field $\zeta$ of $\bar{M}$ from (2.4) is decomposed by

$$
\zeta=P \zeta+\sum_{i=1}^{r} a_{i} \xi_{i}+\sum_{i=1}^{r} b_{i} N_{i}+\sum_{\alpha=r+1}^{n} e_{\alpha} E_{\alpha}
$$

where $a_{i}=\theta\left(N_{i}\right), b_{i}=\theta\left(\xi_{i}\right)$ and $e_{\alpha}=\epsilon_{\alpha} \theta\left(E_{\alpha}\right)$ are smooth functions on $\bar{M}$.

Note 2. Although $S(T M)$ is not unique, it is canonically isomorphic to the factor vector bundle $S(T M)^{*}=T M / \operatorname{Rad}(T M)$ considered by Kupeli [2]. Thus all screen distributions $S(T M)$ are mutually isomorphic. For this reason, the following definition is well defined.

Definition 3.1 (see [6]). One says that $M$ is generic lightlike submanifold of $\bar{M}$ if there exists a screen distribution $S(T M)$ of $M$ such that

$$
J\left(S(T M)^{\perp}\right) \subset S(T M)
$$

Proposition 3.2 (see [3]). Let $M$ be a lightlike hypersurface of an indefinite cosymplectic manifold $\bar{M}$. Then $M$ is a generic lightlike submanifold of $\bar{M}$.

Proposition 3.3 (see [4]). Let $M$ be a 1-lightlike submanifold of codimension 2 of an indefinite cosymplectic manifold $\bar{M}$ such that the coscreen distribution $S\left(T M^{\perp}\right)$ is spacelike. Then $M$ is a generic lightlike submanifold of $\bar{M}$.

Theorem 3.4. Let $M$ be an irrotational generic $r$-lightlike submanifold of an indefinite cosymplectic space form $\bar{M}(c)$. Then one has $c=0$. 
Proof. Assume that $b_{k} \neq 0$ in (3.5). Note that (3.1) implies $\bar{g}\left(J X, \xi_{k}\right)=-\bar{g}\left(X, J \xi_{k}\right)$ for all $X \in$ $\Gamma(T M)$. Then, taking the scalar product with $\xi_{k}$ to (3.4), and using (2.28), we get

$$
\begin{aligned}
4 \sum_{i=1}^{r}\{ & \left.g\left(A_{\xi_{i}}^{*} X, A_{\xi_{k}}^{*} Y\right)-g\left(A_{\xi_{i}}^{*} Y, A_{\xi_{k}}^{*} X\right)\right\} \eta_{i}(Z) \\
= & c\left\{b_{k} g(X, Z) \theta(Y)-b_{k} g(Y, Z) \theta(X)-\bar{g}(J Y, Z) g\left(X, J \xi_{k}\right)\right. \\
& \left.\quad-\bar{g}(J Z, X) g\left(Y, J \xi_{k}\right)+2 \bar{g}(J X, Y) g\left(Z, J \xi_{k}\right)\right\}, \quad \forall X, Y, Z \in \Gamma(T M) .
\end{aligned}
$$

Replace $Z$ by $J \xi_{k}$ and $Y$ by $\xi_{k}$ in the equation and use (3.1), then we have

$$
b_{k}^{2} c g\left(X, J \xi_{k}\right)=0, \quad \forall X \in \Gamma(T M)
$$

because $\eta_{i}\left(J \xi_{k}\right)=0$ by (3.6). Replacing $X$ by $J \xi_{k}$ in this equation, we obtain $b_{k}^{4} c=0$. Since $b_{k} \neq 0$, we have $c=0$.

Assume that $b_{k}=0$. Then, taking the scalar product with $\xi_{k}$ to both sides of (3.4) and using (2.28) and (3.1), we obtain

$$
\begin{aligned}
4 \sum_{i=1}^{r}\left\{g\left(A_{\xi_{i}}^{*} X, A_{\xi_{k}}^{*} Y\right)-g\left(A_{\xi_{i}}^{*} Y, A_{\xi_{k}}^{*} X\right)\right\} \eta_{i}(Z) \\
=c\left\{-\bar{g}(J Y, Z) g\left(X, J \xi_{k}\right)-\bar{g}(J Z, X) g\left(Y, J \xi_{k}\right)\right. \\
\left.\quad+2 \bar{g}(J X, Y) g\left(Z, J \xi_{k}\right)\right\}, \quad \forall X, Y, Z \in \Gamma(T M) .
\end{aligned}
$$

Replace $Z$ by $J N_{k}$ and $Y$ by $\xi_{k}$ in this equation and use (3.1), then we have

$$
c g\left(X, J \xi_{k}\right)=0, \quad \forall X \in \Gamma(T M)
$$

because $\eta_{i}\left(J N_{k}\right)=0$ by (3.6) and $g\left(J \xi_{k}, J N_{k}\right)=1$. Replace $X$ by $J N_{k}$ in this equation, we get $c=0$.

Corollary 3.5. There exist no irrotational generic $r$-lightlike submanifolds $M$ of an indefinite cosymplectic space form $\bar{M}(c)$ with $c \neq 0$.

Proposition 3.6. Let $M$ be an $r$-lightlike submanifold of an indefinite cosymplectic manifold $\bar{M}$. Then the characteristic vector field $\zeta$ does not belong to $\operatorname{Rad}(T M)$ and $\operatorname{ltr}(T M)$.

Proof. Assume that $\zeta$ belongs to $\operatorname{Rad}(T M)$ (or $\operatorname{ltr}(T M))$. Then (3.1) deduces to $\zeta=\sum_{i=1}^{r} a_{i} \xi_{i}$ [or $\zeta=\sum_{i=1}^{r} b_{i} N_{i}$. From this, we have

$$
1=\bar{g}(\zeta, \zeta)=\sum_{i, j=1}^{r} a_{i} a_{j} \bar{g}\left(\xi_{i}, \xi_{j}\right)=0 \quad\left[\text { or } 1=\bar{g}(\zeta, \zeta)=\sum_{i, j=1}^{r} b_{i} b_{j} \bar{g}\left(N_{i}, N_{j}\right)=0\right] .
$$

It is a contradiction. Thus $\zeta$ does not belong to $\operatorname{Rad}(T M)$ and $\operatorname{ltr}(T M)$. 


\section{Generic Lightlike Submanifolds}

If the characteristic vector field $\zeta$ is tangent to $M$, then, by Proposition 3.6, $\zeta$ does not belong to $\operatorname{Rad}(T M)$. This enables one to choose a screen distribution $S(T M)$ which contains $\zeta$. This implies that if $\zeta$ is tangent to $M$, then it belongs to $S(T M)$. Călin also proved this result in his book [11] which Kang et al. [12] and Duggal and Sahin [5, 8] assumed in their papers. We also assumed this result in this paper. In this case, all of the functions $a_{i}, b_{i}$, and $e_{\alpha}$ on $\bar{M}$, defined by (3.5), vanish identically.

Theorem 4.1. Let $M$ be a generic $r$-lightlike submanifold of an indefinite cosymplectic manifold $\bar{M}$. Then $\zeta$ is a parallel vector field on $M$ and $S(T M)$. Furthermore $\zeta$ is conjugate to any vector field on $M$ with respect to $h$ and $h_{i}^{*}$. In particular, $\zeta$ is an asymptotic vector field on $M$.

Proof. Replace $Y$ by $\zeta$ to (2.6) and use (3.3) and $\zeta \in \Gamma(T M)$, we get

$$
\nabla_{X} \zeta+\sum_{j=1}^{r} h_{j}^{\ell}(X, \zeta) N_{j}+\sum_{\beta=r+1}^{n} h_{\beta}^{s}(X, \zeta) E_{\beta}=0, \quad \forall X \in \Gamma(T M) .
$$

Taking the scalar product with $\xi_{i}$ and $E_{\alpha}$ in this equation by turns, we have

$$
\nabla_{X} \zeta=0, \quad h_{i}^{\ell}(X, \zeta)=0, \quad h_{\alpha}^{s}(X, \zeta)=0 .
$$

Thus $\zeta$ is parallel on $M$ and conjugate to any vector field on $M$ with respect to $h$. Replace $P Y$ by $\zeta$ to (2.9) and use (4.2) and $\zeta \in \Gamma(S(T M))$, we have

$$
\nabla_{X}^{*} \zeta+\sum_{j=1}^{r} h_{j}^{*}(X, \zeta) \xi_{j}=0, \quad \forall X \in \Gamma(T M)
$$

Taking the scalar product with $N_{i}$ to this equation we have

$$
\nabla_{X}^{*} \zeta=0, \quad h_{i}^{*}(X, \zeta)=0, \quad \forall X \in \Gamma(T M)
$$

Thus $\zeta$ is also parallel on $S(T M)$ and conjugate to any vector field on $M$ with respect to $h^{*}$. Thus we have our assertions.

Definition 4.2. An $r$-lightlike submanifold $M$ of $\bar{M}$ is said to be totally umbilical [13] if there is a smooth vector field $\mathscr{\ell} \in \Gamma(\operatorname{tr}(T M))$ such that

$$
h(X, Y)=\mathscr{\ell g}(X, Y), \quad \forall X, Y \in \Gamma(T M)
$$

In case $\mathscr{H}=0$, we say that $M$ is totally geodesic.

It is easy to see that $M$ is totally umbilical if and only if, on each coordinate neighborhood $\mathcal{U}$, there exist smooth functions $\mathcal{A}_{i}$ and $\mathcal{B}_{\alpha}$ such that

$$
h_{i}^{\ell}(X, Y)=\mathscr{A}_{i} g(X, Y), \quad h_{\alpha}^{s}(X, Y)=\mathbb{B}_{\alpha} g(X, Y), \quad \forall X, Y \in \Gamma(T M)
$$


Theorem 4.3. Let $M$ be a totally umbilical generic $r$-lightlike submanifold of an indefinite cosymplectic manifold $\bar{M}$. Then $M$ is totally geodesic.

Proof. From (4.2) and (4.6), we obtain

$$
\mathscr{A}_{i} g(X, \zeta)=0, \quad B_{\alpha} g(X, \zeta)=0 .
$$

Replace $X$ by $\zeta$ to this equations and use $g(\zeta, \zeta)=1$, we have $\mathcal{A}_{i}=0$ for all $i$ and $B_{\alpha}=0$ for all $\alpha$. Thus $M$ is totally geodesic.

Definition 4.4. A screen distribution $S(T M)$ of $M$ is said to be totally umbilical [13] in $M$ if, for each locally second fundamental form $h_{i}^{*}$, there exist smooth functions $C_{i}$ on any coordinate neighborhood $\mathcal{U}$ in $M$ such that

$$
h_{i}^{*}(X, P Y)=\mathcal{C}_{i} g(X, Y), \quad \forall X, Y \in \Gamma(T M)
$$

In case $C_{i}=0$ for all $i$, we say that $S(T M)$ is totally geodesic in $M$.

Due to (2.18) and (4.8), we know that $S(T M)$ is totally umbilical in $M$ if and only if each shape operators $A_{N_{i}}$ of $S(T M)$ satisfies

$$
g\left(A_{N_{i}} X, P Y\right)=\mathcal{C}_{i} g(X, P Y), \quad \forall X, Y \in \Gamma(T M),
$$

for some smooth functions $\mathcal{C}_{i}$ on $U \subseteq M$.

Theorem 4.5. Let $M$ be a generic $r$-lightlike submanifold of an indefinite cosymplectic manifold $\bar{M}$ such that $S(T M)$ is totally umbilical in $M$. Then $S(T M)$ is totally geodesic in $M$.

Proof. As $S(T M)$ is totally umbilical in $M$. Replace $Y$ by $\zeta$ to (4.8) and use (4.4), we have $\mathcal{C}_{i} g(X, \zeta)=0$ for all $X \in \Gamma(T M)$. Replace $X$ by $\zeta$ to this equation and use the fact $g(\zeta, \zeta)=1$, we obtain $C_{i}=0$ for all $i$.

From (3.6), the screen distribution $S(T M)$ splits as follows:

$$
S(T M)=\{J(\operatorname{Rad}(T M)) \oplus J(\operatorname{ltr}(T M))\} \oplus_{\text {orth }} J\left(S\left(T M^{\perp}\right)\right) \oplus_{\text {orth }} H_{o}
$$

where $H_{O}$ is a non-degenerate almost complex distribution $H_{o}$ on $M$ with respect to $J$, that is, $J\left(H_{o}\right)=H_{o}$. Thus the general decompositions of $T M$ and $T \bar{M}$ in (2.1) and (2.4) reduce, respectively, to

$$
T M=H \oplus H^{\prime}, \quad T \bar{M}=H \oplus H^{\prime} \oplus \operatorname{tr}(T M),
$$

where $H$ and $H^{\prime}$ are $2 r$ - and $r$-lightlike distributions on $M$ such that

$$
\begin{aligned}
& H=\operatorname{Rad}(T M) \oplus_{\text {orth }} J(\operatorname{Rad}(T M)) \oplus_{\text {orth }} H_{o}, \\
& H^{\prime}=J(\operatorname{ltr}(T M)) \oplus_{\text {orth }} J\left(S\left(T M^{\perp}\right)\right) .
\end{aligned}
$$


In this case, $H$ is an almost complex distribution of $M$ with respect to $J$. Consider the local null vector fields $U_{i}$ and $V_{i}$ on $S(T M)$ and the local nonnull vector field $W_{\alpha}$ on $S(T M)$ defined respectively by

$$
U_{i}=-J N_{i}, \quad V_{i}=-J \xi_{i}, \quad W_{\alpha}=-J E_{\alpha}
$$

Denote by $S$ the projection morphism of $T M$ on $H$ with respect to the decomposition (4.11). Then any vector field $X$ on $M$ is expressed as follows:

$$
\begin{aligned}
& X=S X+\sum_{i=1}^{r} u_{i}(X) U_{i}+\sum_{\alpha=r+1}^{m} w_{\alpha}(X) W_{\alpha} \\
& J X=F X+\sum_{i=1}^{r} u_{i}(X) N_{i}+\sum_{\alpha=r+1}^{m} w_{\alpha}(X) E_{\alpha}
\end{aligned}
$$

where $u_{i}, v_{i}$, and $w_{\alpha}$ are 1 -forms locally defined on $M$ by

$$
u_{i}(X)=g\left(X, V_{i}\right), \quad v_{i}(X)=g\left(X, U_{i}\right), \quad w_{i}(X)=\epsilon_{\alpha} g\left(X, E_{\alpha}\right),
$$

and $F$ is a tensor field of (1,1)-type globally defined on $M$ by

$$
F X=J S X, \quad \forall X \in \Gamma(T M) .
$$

Apply $J$ to (2.6), (2.7), (2.8), and (2.24) and use (4.13) and (4.14), we have

$$
\begin{gathered}
h_{j}^{\ell}\left(X, U_{i}\right)=h_{i}^{*}\left(X, V_{j}\right), \quad h_{i}^{*}\left(X, W_{\alpha}\right)=\epsilon_{\alpha} h_{\alpha}^{s}\left(X, U_{i}\right), \\
h_{j}^{\ell}\left(X, V_{i}\right)=h_{i}^{\ell}\left(X, V_{j}\right), \quad h_{i}^{\ell}\left(X, W_{\alpha}\right)=\epsilon_{\alpha} h_{\alpha}^{s}\left(X, V_{i}\right), \\
h_{i}^{\ell}\left(X, W_{\alpha}\right)=\epsilon_{\alpha} h_{\alpha}^{s}\left(X, V_{i}\right), \quad \epsilon_{\beta} h_{\beta}^{s}\left(X, W_{\alpha}\right)=\epsilon_{\alpha} h_{\alpha}^{s}\left(X, W_{\beta}\right) \\
\nabla_{X} U_{i}=F\left(A_{N_{i}} X\right)+\sum_{j=1}^{r} \tau_{i j}(X) U_{j}+\sum_{\alpha=r+1}^{r} \rho_{i \alpha}(X) W_{\alpha}, \\
\nabla_{X} V_{i}=F\left(A_{\xi_{i}}^{*} X\right)-\sum_{j=1}^{r} \tau_{j i}(X) V_{j}-\sum_{\alpha=r+1}^{r} \epsilon_{\alpha} \phi_{\alpha i}(X) W_{\alpha}+\sum_{j=1}^{r} h_{j}^{\ell}\left(X, \xi_{i}\right) U_{j}, \\
\nabla_{X} W_{\alpha}=F\left(A_{E_{\alpha}} X\right)+\sum_{i=1}^{r} \phi_{\alpha i}(X) U_{i}+\sum_{\beta=r+1}^{n} \sigma_{\alpha \beta}(X) W_{\beta}, \\
\left(\nabla_{X} F\right)(Y)=\sum_{i=1}^{r} u_{i}(Y) A_{N_{i}} X+\sum_{\alpha=r+1}^{n} w_{\alpha}(Y) A_{E_{\alpha}} X-\sum_{i=1}^{r} h_{i}^{\ell}(X, Y) U_{i}-\sum_{\alpha=r+1}^{n} h_{\alpha}^{s}(X, Y) W_{\alpha} .
\end{gathered}
$$


Theorem 4.6. Let $M$ be a generic $r$-lightlike submanifold of an indefinite cosymplectic manifold $\bar{M}$. Then $\mathrm{H}$ is integrable if and only if

$$
h(X, F Y)=h(F X, Y), \quad \forall X, Y \in \Gamma(H) .
$$

Moreover, if $M$ is totally umbilical, then $H$ is a parallel distribution on $M$.

Proof. Take $X, Y \in \Gamma(H)$. Then we have $F Y=J Y \in \Gamma(H)$. Apply $\bar{\nabla}_{X}$ to $F Y=J Y$ and use (2.6), (3.2), (4.13), and (4.14), we have

$$
\begin{aligned}
& h_{i}^{\ell}(X, F Y)=g\left(\nabla_{X} Y, V_{i}\right), \quad h_{\alpha}^{s}(X, F Y)=\epsilon_{\alpha} g\left(\nabla_{X} Y, W_{\alpha}\right), \\
& \left(\nabla_{X} F\right)(Y)=-\sum_{i=1}^{r} h_{i}^{\ell}(X, Y) U_{i}-\sum_{\alpha=r+1}^{m} h_{\alpha}^{s}(X, Y) W_{\alpha} .
\end{aligned}
$$

By directed calculations from two equations of (4.25), we have

$$
h(X, F Y)-h(F X, Y)=\sum_{i=1}^{r} g\left([X, Y], V_{i}\right) N_{i}+\sum_{\alpha=r+1}^{m} \epsilon_{\alpha} g\left([X, Y], W_{\alpha}\right) E_{\alpha}
$$

If $H$ is an integrable distribution on $M$, then we have $[X, Y] \in \Gamma(H)$ for any $X, Y \in \Gamma(H)$. This implies $g\left([X, Y], V_{i}\right)=g\left([X, Y], W_{\alpha}\right)=0$ for all $i$ and $\alpha$. Therefore we obtain $h(X, F Y)=$ $h(F X, Y)$ for all $X, Y \in \Gamma(H)$. Conversely if $h(X, F Y)=h(F X, Y)$ for all $X, Y \in \Gamma(H)$, then we have $g\left([X, Y], V_{i}\right)=g\left([X, Y], W_{\alpha}\right)=0$ for all $i$ and $\alpha$. This implies $[X, Y] \in \Gamma(H)$ for all $X, Y \in \Gamma(H)$. Thus $H$ is an integrable distribution of $M$.

If $M$ is totally umbilical, from Theorem 4.3 and (4.25), we have

$$
g\left(\nabla_{X} Y, V_{i}\right)=g\left(\nabla_{X} Y, W_{\alpha}\right)=0, \quad \forall i, \alpha .
$$

This implies $\nabla_{X} Y \in \Gamma(H)$ for all $X, Y \in \Gamma(H)$. Thus $H$ is a parallel distribution on $M$.

Theorem 4.7. Let $M$ be a generic $r$-lightlike submanifold of an indefinite cosymplectic manifold $\bar{M}$. Then $F$ is parallel on $H$ with respect to $\nabla$ if and only if $H$ is a parallel distribution on $M$.

Proof. Assume that $F$ is parallel on $H$ with respect to $\nabla$. For any $X, Y \in \Gamma(H)$, we have $\left(\nabla_{X} F\right) Y=0$. Taking the scalar product with $V_{k}$ and $W_{\beta}$ to $(4.26)$ with $\left(\nabla_{X} F\right) Y=0$, we have $h_{k}^{\ell}(X, Y)=0$ and $h_{\beta}^{s}(X, Y)=0$ for all $X, Y \in \Gamma(H)$ and for each $k$ and $\beta$, respectively. From (4.25), we have $g\left(\nabla_{X} Y, V_{i}\right)=0$ and $g\left(\nabla_{X} Y, W_{\alpha}\right)=0$. This implies $\nabla_{X} Y \in \Gamma(H)$ for all $X, Y \in$ $\Gamma(H)$. Thus $H$ is a parallel distribution on $M$.

Conversely, if $H$ is parallel on $M$, from (4.25) we have

$$
h_{i}^{\ell}(X, F Y)=0, \quad h_{\alpha}^{s}(X, F Y)=0, \quad \forall X, Y \in \Gamma(H) .
$$

For any $Y \in \Gamma(H)$, we show that $F^{2} Y=J^{2} Y=-Y+\theta(Y) \zeta$. Replace $Y$ by $F Y$ to the equations and use (4.2), we have $h_{i}^{\ell}(X, Y)=0$ and $h_{\alpha}^{s}(X, Y)=0$ for any $X, Y \in \Gamma(H)$. Thus $F$ is parallel on $H$ with respect to $\nabla$ by (4.25). 
Theorem 4.8. Let $M$ be a generic $r$-lightlike submanifold of an indefinite cosymplectic manifold $\bar{M}$. If $F$ is parallel on $T M$ with respect to $\nabla$, then $H$ is a parallel distribution on $M$ and $M$ is locally a product manifold $M^{r} \times M^{n-r} \times M^{m-n}$, where $M^{r}, M^{n-r}$, and $M^{m-n}$ are leafs of $J(\operatorname{ltr}(T M))$, $J\left(S\left(T M^{\perp}\right)\right)$ and $H$, respectively.

Proof. Assume that $F$ is parallel on $T M$ with respect to $\nabla$. Then $F$ is parallel on $H$ with respect to $\nabla$. By Theorem 4.7, $H$ is a parallel distribution on $M$. Apply the operator $F$ to (4.23) with $\left(\nabla_{X} F\right) Y=0$, we have

$$
\sum_{i=1}^{r} u_{i}(Y) F\left(A_{N_{i}} X\right)+\sum_{\alpha=r+1}^{n} e_{\alpha}(Y) F\left(A_{E_{\alpha}} X\right)=0, \quad \forall X, Y \in \Gamma(T M),
$$

due to $F U_{i}=F W_{\alpha}=0$ for all $i$ and $\alpha$. Replace $Y$ by $U_{k}$ and $W_{\beta}$ to this equation by turns and use (4.15), we have $F\left(A_{N_{i}} X\right)=0$ and $F\left(A_{E_{\alpha}} X\right)=0$. Taking the scalar product with $W_{\beta}$ and $N_{k}$ to $(4.23)$ with $\left(\bar{\nabla}_{X} F\right) Y=0$ by turns, we have

$$
\begin{aligned}
& h_{\alpha}^{s}(X, Y)=\sum_{i=1}^{r} u_{i}(Y) w_{\alpha}\left(A_{N_{i}} X\right)+\sum_{\beta=r+1}^{m} w_{\beta}(Y) w_{\alpha}\left(A_{E_{\beta}} X\right), \\
& \sum_{i=1}^{r} u_{i}(Y) g\left(A_{N_{i}} X, N_{k}\right)+\sum_{\alpha=r+1}^{m} w_{\alpha}(Y) g\left(A_{E_{\alpha}} X, N_{k}\right)=0,
\end{aligned}
$$

for all $X, Y \in \Gamma(T M)$. Replace $Y$ by $\xi_{j}$ to (4.31), we get $\phi_{\alpha j}(X)=0$ due to (2.23). Also replace $Y$ by $W_{\beta}$ to (4.32), we have $\rho_{k \beta}(X)=0$ due to (2.17). From this results, (4.11) and (4.14), we get

$$
\nabla_{X} U_{i}=\sum_{j=1}^{r} \tau_{i j}(X) U_{j}, \quad \nabla_{X} W_{\alpha}=\sum_{\beta=r+1}^{m} \sigma_{\alpha \beta}(X) W_{\beta}, \quad \forall X \in \Gamma(T M)
$$

Thus $J(\operatorname{ltr}(T M))$ and $J\left(S\left(T M^{\perp}\right)\right)$ are also parallel distributions on $M$. By the decomposition theorem of de Rham [14], we show that $M=M^{r} \times M^{n-r} \times M^{m-n}$, where $M^{r}, M^{n-r}$, and $M^{m-n}$ are some leafs of $J(\operatorname{ltr}(T M)), J\left(S\left(T M^{\perp}\right)\right)$ and $H$, respectively.

Theorem 4.9. Let $M$ be a generic $r$-lightlike submanifold of an indefinite cosymplectic manifold $\bar{M}$. One has the following assertions.

(i) If each $V_{i}$ is parallel with respect to $\nabla$, then $\tau_{i j}=\phi_{\alpha i}=h^{\ell}\left(X, \xi_{i}\right)=0$. In this case $M$ is irrotational. Moreover, one has

$$
A_{\xi_{i}}^{*} X=\sum_{j=1}^{r} u_{j}\left(A_{\xi_{i}}^{*} X\right) U_{j}+\sum_{\alpha=r+1}^{m} w_{\alpha}\left(A_{\xi_{i}}^{*} X\right) W_{\alpha}, \quad \forall X \in \Gamma(T M) .
$$

(ii) If each $U_{i}$ is parallel with respect to $\nabla$, then $\tau_{i j}=\rho_{i \alpha}=0$ and

$$
A_{N_{i}} X=\sum_{j=1}^{r} u_{j}\left(A_{N_{i}} X\right) U_{j}+\sum_{\alpha=r+1}^{m} w_{\alpha}\left(A_{N_{i}} X\right) W_{\alpha}, \quad \forall X \in \Gamma(T M) .
$$


(iii) If each $W_{\alpha}$ is parallel with respect to $\nabla$, then $\phi_{\alpha i}=0$ and

$$
A_{E_{\alpha}} X=\sum_{i=1}^{r} u_{i}\left(A_{E_{\alpha}} X\right) U_{i}+\sum_{\beta=r+1}^{m} w_{\beta}\left(A_{E_{\alpha}} X\right) W_{\beta}, \quad \forall X \in \Gamma(T M)
$$

Moreover, if all of $V_{i}, U_{i}$, and $W_{\alpha}$ are parallel on TM with respect to $\nabla$, then $S(T M)$ is totally geodesic in $M$ and $\tau_{i j}=\phi_{\alpha i}=\rho_{i \alpha}=0$ on $\Gamma(T M)$. In this case, each null transversal vector fields $N_{i}$ of $M$ is a constant on $M$.

Proof. If $V_{i}$ is parallel with respect to $\nabla$, then, taking the scalar product with $U_{k}, W_{\beta}$, and $V_{k}$ to (4.21) by turns, we have $\tau_{k i}(X)=0, \phi_{\beta i}(X)=0$ and $h_{k}^{\ell}\left(X, \xi_{i}\right)=0$, respectively. Thus $M$ is irrotational. We have $F\left(A_{\xi_{i}}^{*} X\right)=0$ for all $X \in \Gamma(T M)$. From this result and (4.14), we obtain

$$
J\left(A_{\xi_{i}}^{*} X\right)=\sum_{j=1}^{r} u_{j}\left(A_{\xi_{i}}^{*} X\right) N_{j}+\sum_{\alpha=r+1}^{m} w_{\alpha}\left(A_{\xi_{i}}^{*} X\right) E_{\alpha}
$$

Apply $J$ to this equation and use $\theta\left(A_{\xi}^{*} X_{i}\right)=0$, we obtain (i). In a similar way, by using (4.13), (4.14), (4.20), and (4.22), we have (ii) and (iii).

Assume that all of $V, U$ and $W$ are parallel on $T M$ with respect to $\nabla$. Substituting the equation of (i) into (4.17)-1, we have

$$
u_{j}\left(A_{N_{i}} X\right)=v_{i}\left(A_{\xi_{j}}^{*} X\right)=g\left(A_{\xi_{j}}^{*} X, U_{i}\right)=0, \quad \forall X \in \Gamma(T M) .
$$

Also, substituting the equation of (iii) into (4.17)-2, we have

$$
w_{\alpha}\left(A_{N_{i}} X\right)=\epsilon_{\alpha} v_{i}\left(A_{E_{\alpha}} X\right)=g\left(A_{E_{\alpha}} X, U_{i}\right)=0, \quad \forall X \in \Gamma(T M)
$$

From the last two equations and the equation of (ii), we see that $A_{N_{i}} X=0$ for all $X \in \Gamma(T M)$. From this and (2.18) we see that $S(T M)$ is totally geodesic in $M$ and all 1-forms $\tau_{i j}, \phi_{\alpha i}$, and $\rho_{i \alpha}$, defined by (2.7) and (2.8), vanish identically. Using the results and (2.7), we show that $N$ is a constant on $M$.

Theorem 4.10. Let $M$ be a totally umbilical generic $r$-lightlike submanifold of an indefinite cosymplectic manifold $\bar{M}$ such that $S(T M)$ is totally umbilical. Then $M$ is locally a product manifold $M^{r} \times M^{s} \times M^{t}$, where $M^{r}, M^{s}$, and $M^{t}$ are some leafs of $\operatorname{Rad}(T M), H_{o}^{\perp}=\operatorname{Span}\left\{V_{i}, U_{i}, W_{\alpha}\right\}$ and $H_{o}$, respectively, and $s=n+r, t=m-n-2 r$.

Proof. By Theorem 4.6, $H$ is a parallel distribution $M$. Thus, for all $X, Y \in \Gamma\left(H_{0}\right)$, we have $\nabla_{X} Y \in \Gamma(H)$. From (2.9) and (4.26), we have

$$
\begin{aligned}
h_{i}^{*}(X, F Y) & =g\left(\nabla_{X} F Y, N_{i}\right)=g\left(\left(\nabla_{X} F\right) Y+F\left(\nabla_{X} Y\right), N_{i}\right) \\
& =g\left(F\left(\nabla_{X} Y\right), N_{i}\right)=-g\left(\nabla_{X} Y, J N_{i}\right)=g\left(\nabla_{X} Y, U_{i}\right),
\end{aligned}
$$


due to $F Y \in \Gamma\left(H_{o}\right)$. If $S(T M)$ is totally umbilical in $M$, then we have $h_{i}^{*}=0$ due to Theorem 4.5. By (2.9) and (4.40), we get

$$
g\left(\nabla_{X} Y, N_{i}\right)=0, \quad g\left(\nabla_{X} Y, U_{i}\right)=0, \quad \forall X \in \Gamma(T M), \forall Y \in \Gamma\left(H_{o}\right)
$$

These results and (4.25) imply $\nabla_{X} Y \in \Gamma\left(H_{o}\right)$ for all $X, Y \in \Gamma\left(H_{o}\right)$. Thus $H_{o}$ is a parallel distribution on $S(T M)$ and $T M=H_{o} \oplus_{\text {orth }} H_{o}^{\perp}$, where $H_{o}^{\perp}=\operatorname{Span}\left\{\xi_{i}, V_{i}, U_{i}, W_{\alpha}\right\}$. By Theorems 4.3 and 4.5, we have $h_{i}^{\ell}=h_{\alpha}^{s}=A_{N_{i}}=\phi_{\alpha i}=0$ and $A_{E_{\alpha}} X=\sum_{i=1}^{r} \rho_{i \alpha}(X) \xi_{i}$. Thus (2.10) and (4.20) (4.22) deduce, respectively, to

$$
\begin{gathered}
\nabla_{X} U_{i}=\sum_{j=1}^{r} \tau_{i j}(X) U_{j}+\sum_{\alpha=r+1}^{m} \rho_{i \alpha}(X) W_{\alpha}, \\
\nabla_{X} V_{i}=-\sum_{j=1}^{r} \tau_{j i}(X) V_{j}+\sum_{j=1}^{r} h_{j}^{\ell}\left(X, \xi_{i}\right) U_{j}, \\
\nabla_{X} W_{\alpha}=-\sum_{i=1}^{r} \rho_{i \alpha}(X) V_{j}+\sum_{\beta=r+1}^{m} \sigma_{\alpha \beta}(X) W_{\beta}, \\
\nabla_{X} \xi=-\sum_{j=1}^{r} \tau_{j i}(X) \xi_{j}, \quad \forall X \in \Gamma\left(H_{o}^{\perp}\right) .
\end{gathered}
$$

Thus $H_{o}^{\perp}$ is also a parallel distribution on $M$. Thus we have $M=M^{r} \times M^{s} \times M^{t}$, where $M^{r}$, $M^{s}$, and $M^{t}$ are some leafs of $\operatorname{Rad}(T M), H_{o}^{\perp}=\operatorname{Span}\left\{V_{i}, U_{i}, W_{\alpha}\right\}$ and $H_{o}$, respectively, and $s=n+r, t=m-n-2 r$.

\section{Acknowledgment}

The authors are thankful to the referee for making various constructive suggestions and corrections towards improving the final version of this paper.

\section{References}

[1] A. Bejancu and K. L. Duggal, "Lightlike submanifolds of semi-Riemannian manifolds," Acta Applicandae Mathematicae, vol. 38, no. 2, pp. 197-215, 1995.

[2] D. N. Kupeli, Singular Semi-Riemannian Geometry, vol. 366 of Mathematics and Its Applications, Kluwer Academic Publishers Group, Dordrecht, The Netherlands, 1996.

[3] D. H. Jin, "Geometry of lightlike hypersurfaces of an indefinite cosymplectic," Communications of the Korean Mathematical Society. In press.

[4] D. H. Jin, "Half lightlike submanifolds of an indefinite cosymplectic," Bulletin of the Korean Mathematical Society. In press.

[5] K. L. Duggal and B. Sahin, "Generalized Cauchy-Riemann lightlike submanifolds of Kaehler manifolds," Acta Mathematica Hungarica, vol. 112, no. 1-2, pp. 107-130, 2006.

[6] D. H. Jin, "Generic lightlike submanifolds of an indefinite Sasakian manifold," Indian Journal of Pure and Applied Mathematics. In press.

[7] T. Takahashi, "Sasakian manifold with pseudo-Riemannian metric," The Tohoku Mathematical Journal, vol. 21, pp. 271-290, 1969. 
[8] K. L. Duggal and B. Sahin, "Lightlike submanifolds of indefinite Sasakian manifolds," International Journal of Mathematics and Mathematical Sciences, vol. 2007, Article ID 57585, 21 pages, 2007.

[9] D. E. Blair, Riemannian Geometry of Contact and Symplectic Manifolds, vol. 203 of Progress in Mathematics, Birkhäuser, Boston, Mass, USA, 2002.

[10] G. D. Ludden, "Submanifolds of cosymplectic manifolds," Journal of Differential Geometry, vol. 4, pp. 237-244, 1970.

[11] C. Călin, Contributions to geometry of CR-submanifold, thesis, University of Iasi, Romania, 1998.

[12] T. H. Kang, S. D. Jung, B. H. Kim, H. K. Pak, and J. S. Pak, "Lightlike hypersurfaces of indefinite Sasakian manifolds," Indian Journal of Pure and Applied Mathematics, vol. 34, no. 9, pp. 1369-1380, 2003.

[13] K. L. Duggal and A. Bejancu, Lightlike Submanifolds of Semi-Riemannian Manifolds and Applications, vol. 364 of Mathematics and its Applications, Kluwer Academic Publishers Group, Dordrecht, The Netherlands, 1996.

[14] G. de Rham, "Sur la reductibilité d'un espace de Riemann," Commentarii Mathematici Helvetici, vol. 26, pp. 328-344, 1952. 


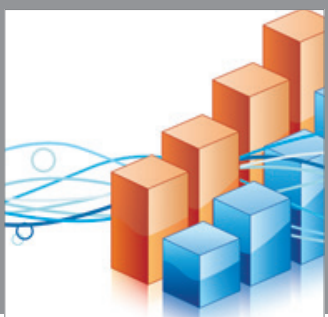

Advances in

Operations Research

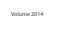

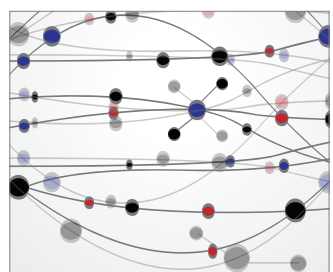

\section{The Scientific} World Journal
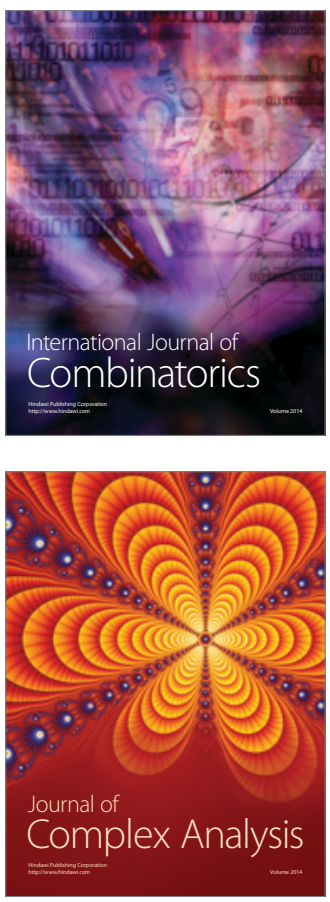

International Journal of

Mathematics and

Mathematical

Sciences
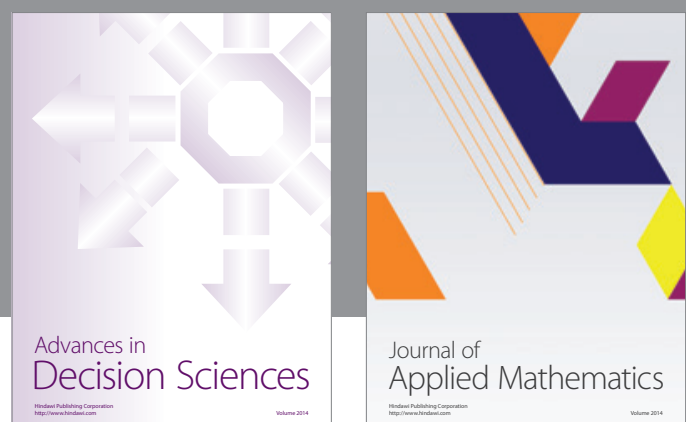

Journal of

Applied Mathematics
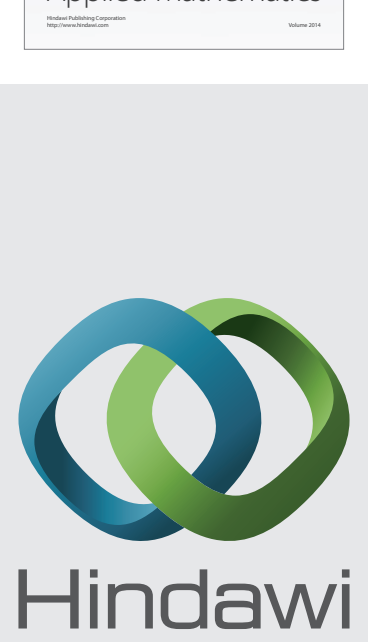

Submit your manuscripts at http://www.hindawi.com
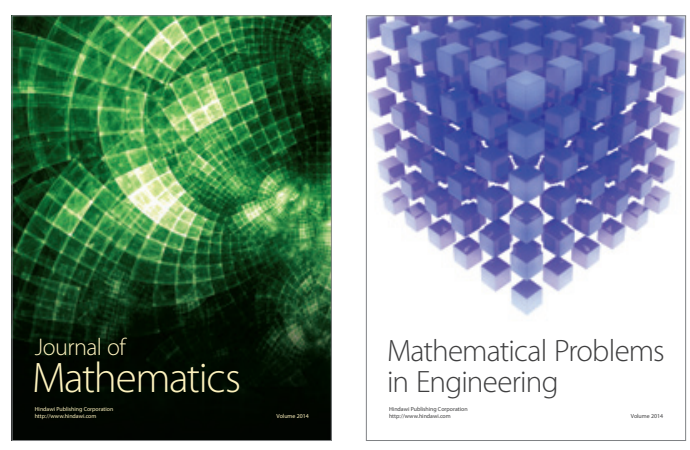

Mathematical Problems in Engineering
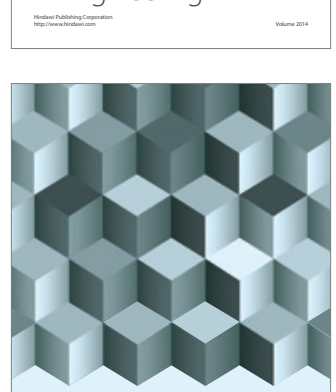

Journal of

Function Spaces
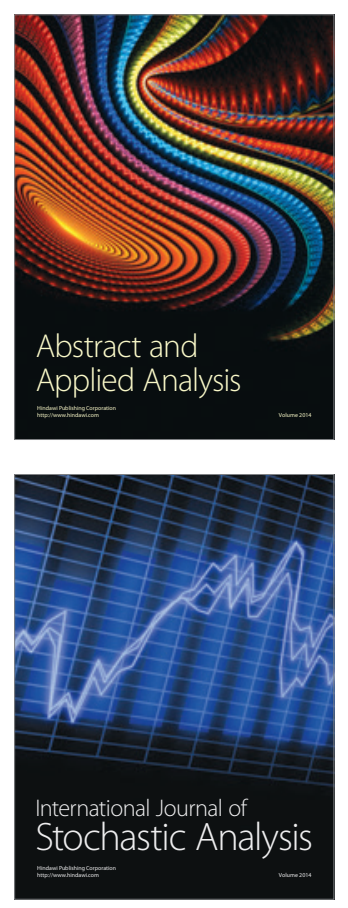

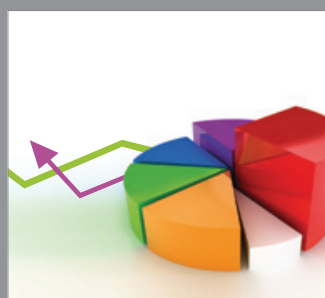

ournal of

Probability and Statistics

Promensencen
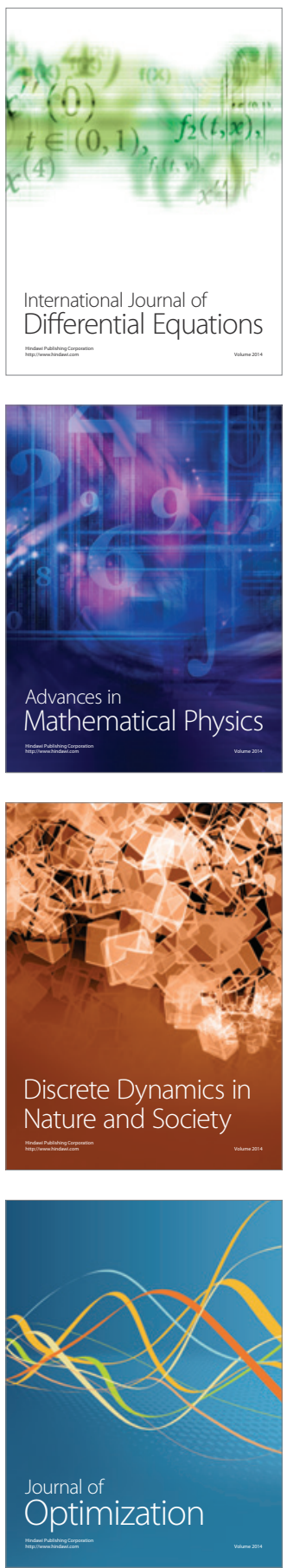